\title{
Hadron Production Measurements for Neutrino Experiments with NA61/SHINE
}

\section{Scott Johnson*}

University of Colorado Boulder (US)

E-mail: scott.robert.johnson@cern.ch

\begin{abstract}
Particle spectra have been obtained from interactions of $31 \mathrm{GeV} / \mathrm{c}$ protons and pions on a thin carbon target with data taken in 2009 and interactions of protons on a T2K replica target with data taken in 2009 and 2010 by NA61/SHINE. These spectra include multiplicities of $\pi^{+}, \pi^{-}$, $K^{+}, K^{-}$, protons, $\Lambda^{0}$ and $K_{s}^{0}$. The results from the 2009 proton data have recently been published in two papers - one for the thin target results and one for the replica target results. Currently, analyses of the 2010 replica target data and 2009 pion on thin target data are ongoing. The charged particle spectra were obtained using $\mathrm{dE} / \mathrm{dx}$ and timing information from NA61/SHINE's TPC and ToF systems, while the neutral particle spectra were obtained using vertex analyses. The thin target measurements have already been used to tune the $\mathrm{T} 2 \mathrm{~K}$ beam MC and reduce the uncertainties on the T2K neutrino flux prediction. Additionally, a method has been developed to apply the $\mathrm{T} 2 \mathrm{~K}$ replica target measurements to the $\mathrm{T} 2 \mathrm{~K}$ beam MC tuning, which is expected to further constrain the T2K neutrino flux predictions especially with the addition of the larger 2010 data set. Having demonstrated its usefulness for improving T2K's results, NA61/SHINE is poised to study the hadron interactions important for Fermilab neutrino experiments. The results from the 2009 pion carbon data will be useful for understanding pion reinteractions in the LBNF and NuMI beamlines. For a complete hadron interaction study, an extensive data program with proton and pion beams at higher energies is planned at NA61/SHINE over the next couple of years.
\end{abstract}

38th International Conference on High Energy Physics

3-10 August 2016

Chicago, USA

${ }^{*}$ Speaker. 


\section{The NA61/SHINE Experiment}

The NA61/SHINE (SPS Heavy Ion and Neutrino Experiment) collaboration consists of about 140 physicists from 14 countries and 28 institutions. The NA61/SHINE detector is a large acceptance hadron spectrometer located at the North Area of CERN [1]. NA61/SHINE receives a secondary beam extracted from the Super Proton Syncrotron (SPS), which is capable of delivering hadrons and heavy ions with energies ranging from 13 to $350 \mathrm{GeV} / \mathrm{c}^{2}$. NA61/SHINE has run with a large variety of targets including a liquid hydrogen target, thin targets of various nuclear material and a T2K replica target. With this large set of capabilities, NA61/SHINE sustains a broad physics program including nuclear physics, neutrino physics and cosmic ray physics.

The NA61/SHINE TPC system currently consists of 5 TPCs, which allow for tracking of charged particles and measuring energy loss. Two vertex TPCs (VTPC1 and VTPC2) are each encased by a superconducting vertex magnet capable of generating a $9 \mathrm{Tm}$ magnetic field. The gap TPC (GTPC) sits in between the two VTPCs and improves the forward acceptance of the detector. Two main TPCs (MTPC right and left) provide the bulk of the $\mathrm{dE} / \mathrm{dx}$ measurement. The $\mathrm{dE} / \mathrm{dx}$ resolution of the TPC system is $\frac{\sigma_{d E / d x}}{\langle d E / d x\rangle} \approx .04$.

NA61/SHINE has three Time of Flight (ToF) subdetectors: ToF Forward (ToF-F), ToF Left (ToF-L) and ToF Right (ToF-R). The resolution of about $100 \mathrm{ps}$ allows for improved particle identification in the momentum range 1 to $10 \mathrm{GeV} / \mathrm{c}$.

Two additional TPCs (Forward TPC 1 and 2) will be installed and implemented before the Fermilab neutrino physics program is finished. The first will be placed between VTPC 2 and the MTPCs and, the second will be placed downstream of the MTPCs. These detectors will greatly improve the forward acceptance, which is critical in understanding forward proton production and pion rescattering in neutrino beamlines.

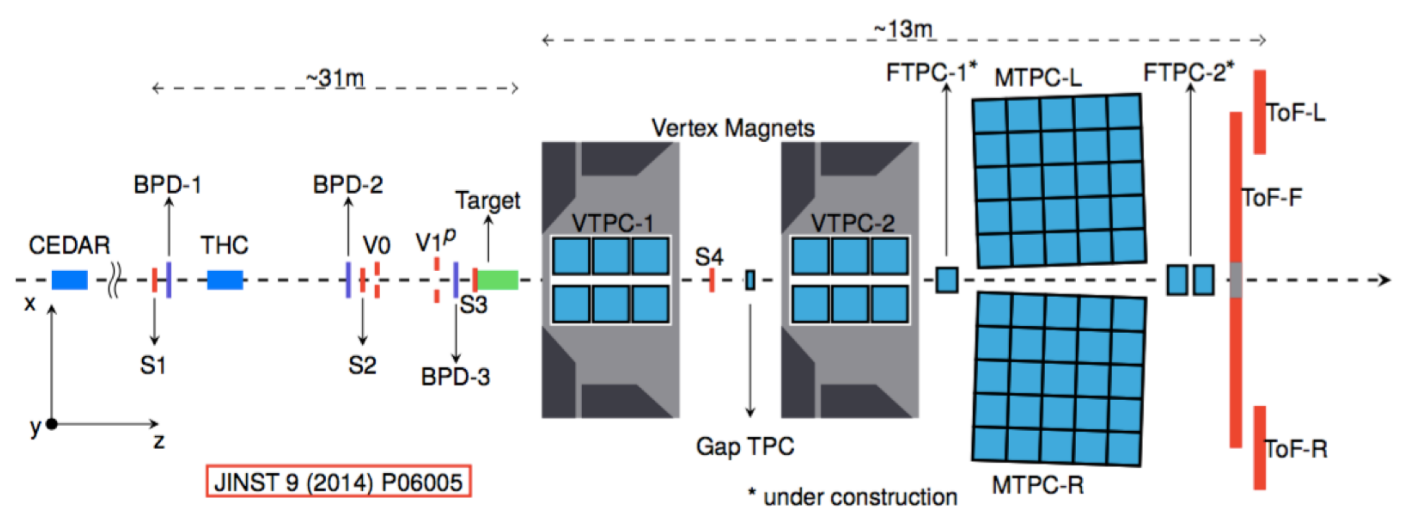

Figure 1: Schematic of the NA61/SHINE detector.

\section{Data for the T2K Experiment}

The neutrino physics program focusses on interactions that are important for understanding the neutrino flux in long baseline neutrino experiments. Interactions of $31 \mathrm{GeV} / \mathrm{c}$ protons with a 2 $\mathrm{cm}$ carbon target were recorded in 2007 and 2009. The measurements from this data have already 
been used to improve T2K's neutrino flux prediction. Results can be found in [2], [3] and [4]. The measurements include differential cross sections binned in production angle $(\theta)$ and momentum ( $p$ ) for seven particle species $\left(\pi^{+}, \pi^{-}, K^{+}, K^{-}, K_{s}^{0}\right.$, and $\left.\Lambda_{0}\right)$. Figure 2 shows a sample of these results.
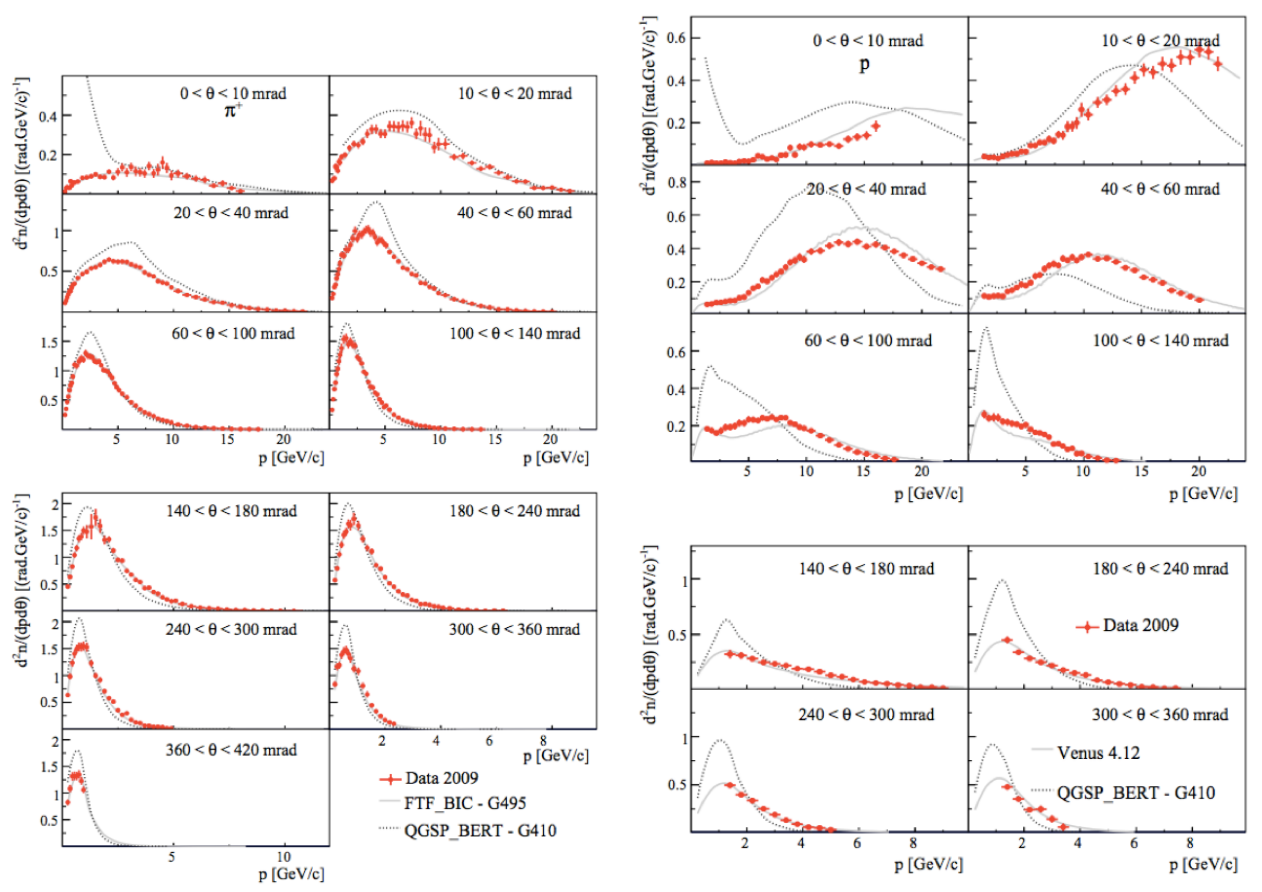

Figure 2: Multiplicity spectra of $\pi^{+}$and protons from the 2009 thin target analysis.

Additionally, NA61/SHINE recorded interactions of $31 \mathrm{GeV} / \mathrm{c}$ protons with a T2K replica target in 2007, 2009 and 2010. Differential cross section measurements from the replica target data are further binned according to where the produced particles exit the target along the beam direction. Differential cross sections of $\pi^{+}$and $\pi^{-}$were measured from the 2007 [5] and 2009 data sets[6].

Analysis of the 2010 T2K replica target data is ongoing. This data run was taken with improved statistics and with an upgraded detector. The extra data enables the analysis of $K^{+}$and $K^{-}$ as well as $\pi^{+}$and $\pi^{-}$. A method has been developed to tune the T2K beam MC with this replica target data in order to further constrain T2K's flux prediction. The results from the 2010 dataset are necessary to achieve T2K's design goal of reducing the neutrino flux uncertainty to less than $5 \%$.

\section{Data for FNAL Neutrino Experiments}

During the 2009 T2K data run, a secondary trigger recorded interactions of mostly pions at 31 $\mathrm{GeV} / \mathrm{c}$ with the carbon target. These interactions are important for understanding pion rescattering in Fermilab neutrino beams. Multiplicity spectra of $\pi^{+}$and $\pi^{-}$are currently being analyzed from these interactions. A sample of these multiplicites is shown in Figure 3. The normalization of these spectra has been obscured due to the difficulty in correctly normalizing the distributions. Analysis of the 2015 cross section data will help resolve this normalization issue. 

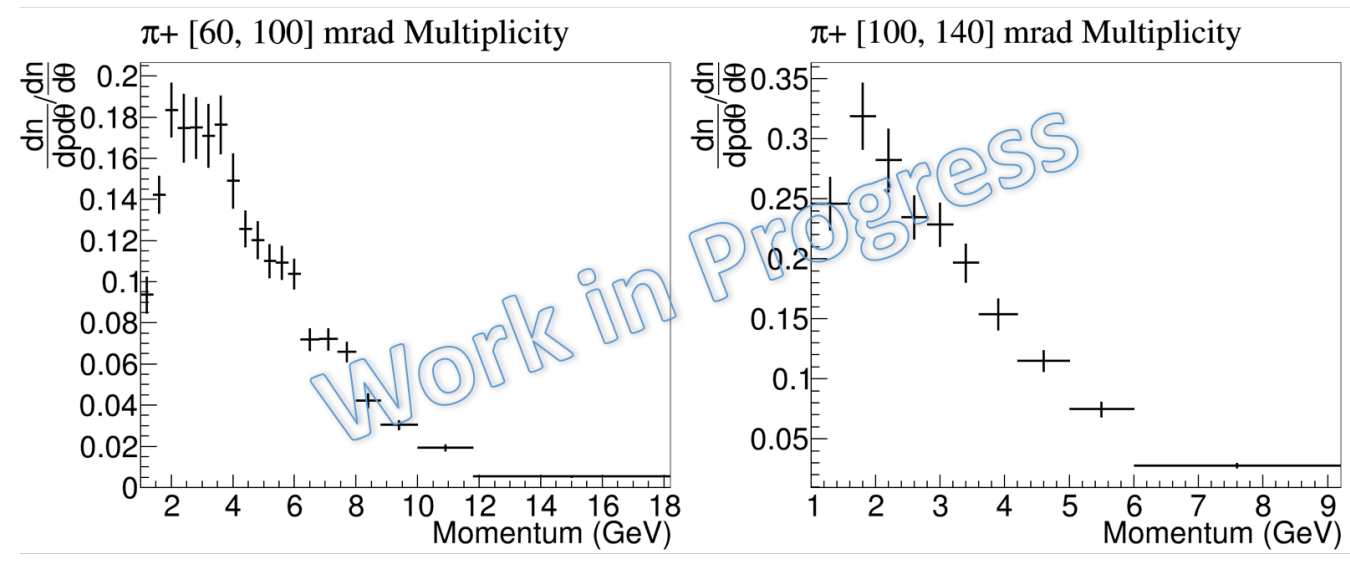

Figure 3: Preliminary results from the analysis of $2009 \pi C$ data.

NA61/SHINE has begun an extensive physics program to benefit the neutrino experiments on the LBNF and NuMI beamlines at Fermilab (DUNE, MINER $\vee$ A, MINOS+ and NO $\vee$ A). During data runs in 2012 and 2015, dedicated total cross section data were taken for a variety of interactions. Interactions of incident protons, $\pi^{+}$and $K^{+}$at momenta of 31,60 and $120 \mathrm{GeV} / \mathrm{c}$ on carbon and aluminum targets were recorded. These data were taken with reduced or no magnetic field, so the data is not suitable for performing spectra measurements. However, measurements of the total inelastic and production cross sections for each interaction, which are important for neutrino experiments, are currently being analyzed.

In the fall of 2016, NA61/SHINE took spectra data for FNAL neutrino experiments with a variety of interactions. These interactions include combinations of $\pi^{+}$and protons at $60 \mathrm{GeV} / \mathrm{c}$ and $120 \mathrm{GeV} / \mathrm{c}$ with carbon, aluminum and beryllium targets. These data are currently being calibrated and will be analyzed in a manner similar to the T2K proton-Carbon data. Another data run is now being planned for the fall of 2017 with more interactions and detector upgrades.

\section{References}

[1] NA61 Collaboration (Abgrall, N. et al.) JINST 9 (2014) P06005 arXiv:1401.4699 [physics.ins-det] CERN-PH-EP-2014-003

[2] NA61/SHINE Collaboration (Abgrall, N et al.) Phys.Rev. C84 (2011) 034604 arXiv:1102.0983 [hep-ex] CERN-PH-EP-2011-005

[3] NA61/SHINE Collaboration (Abgrall, N. et al.) Phys.Rev. C85 (2012) 035210 arXiv:1112.0150 [hep-ex]

[4] NA61/SHINE Collaboration, Abgrall, N., Aduszkiewicz, A. et al. Eur. Phys. J. C (2016) 76: 84. doi:10.1140/epjc/s10052-016-3898-y

[5] NA61/SHINE Collaboration (Abgrall, N. et al.) Nucl.Instrum.Meth. A701 (2013) 99-114 arXiv:1207.2114 [hep-ex] CERN-PH-EP-2012-188

[6] Abgrall, N., Aduszkiewicz, A., Ajaz, M. et al. Eur. Phys. J. C (2016) 76: 617. doi:10.1140/epjc/s10052-016-4440-y 